\title{
ALMA imaging of the CO snowline of the HD 163296 disk with $\mathrm{DCO}^{+\star}$
}

\author{
G. S. Mathews ${ }^{1}$, P. D. Klaassen ${ }^{1}$, A. Juhász ${ }^{1}$, D. Harsono ${ }^{1,2}$, E. Chapillon ${ }^{3}$, E. F. van Dishoeck ${ }^{1,4}$, D. Espada ${ }^{5,6}$, \\ I. de Gregorio-Monsalvo ${ }^{7,8}$, A. Hales ${ }^{8}$, M. R. Hogerheijde ${ }^{1}$, J. C. Mottram ${ }^{1}$, M. G. Rawlings ${ }^{9}$, \\ S. Takahashi ${ }^{8,5,3}$, and L. Testi ${ }^{7,10}$ \\ ${ }^{1}$ Leiden Observatory, Leiden University, PO Box 9513, 2300 RA Leiden, The Netherlands \\ e-mail: gmathews@strw.leidenuniv.nl \\ 2 SRON Netherlands Institute for Space Research, PO Box 800, 9700 AV Groningen, The Netherlands \\ 3 Academia Sinica Institute of Astronomy and Astrophysics (ASIAA), PO Box 23-141, 10617 Taipei, Taiwan \\ 4 Max-Planck-Institut für Extraterrestrische Physik, Giessenbachstrasse 1, 85748 Garching, Germany \\ 5 National Astronomical Observatory of Japan (NAOJ), 2-21-1 Osawa, Mitaka, 181-8588 Tokyo, Japan \\ ${ }^{6}$ NAOJ Chile Observatory \\ 7 European Southern Observatory, Karl Schwarzschild Str 2, 85748 Garching bei München, Germany \\ 8 Joint ALMA Observatory (JAO), Alonso de Cordova 3107, Vitacura, Santiago, Chile \\ 9 National Radio Astronomical Observatory (NRAO), 520 Edgemont Road, Charlottesville, VA 22903, USA \\ 10 INAF - Osservatorio Astrofisico di Arcetri, Largo E. Fermi 5, 50125 Firenze, Italy
}

Received 29 March 2013 / Accepted 11 July 2013

\section{ABSTRACT}

Context. The high spatial resolution and line sensitivity of the Atacama Large Millimeter/submillimeter Array (ALMA) opens the possibility of resolving emission from molecules in large samples of circumstellar disks. With an understanding of the conditions under which these molecules can have high abundance, they can be used as direct tracers of distinct physical regions. In particular, $\mathrm{DCO}^{+}$is expected to have an enhanced abundance within a few Kelvin of the CO freezeout temperature of $19 \mathrm{~K}$, making it a useful probe of the cold disk midplane.

Aims. We aim to use line emission from $\mathrm{DCO}^{+}$to directly resolve the $\mathrm{CO}$ "snowline" - the region at which the gas-phase $\mathrm{CO}$ abundance drops due to freezeout - and determine the temperature boundaries of the region of $\mathrm{DCO}^{+}$emission in the HD 163296 disk. This will serve as a test of deuteration models based on enhanced formation of the parent molecule $\mathrm{H}_{2} \mathrm{D}^{+}$and a direct probe of midplane disk structure and ionization.

Methods. We compare ALMA line observations of HD 163296 to a grid of models based on the best fit physical model of Qi et al. (2011, ApJ, 740, 84). We vary the upper- and lower-limit temperatures of the region in which $\mathrm{DCO}^{+}$is present as well as the abundance of $\mathrm{DCO}^{+}$in order to fit channel maps of the $\mathrm{DCO}^{+} J=5-4$ line. To determine the abundance enhancement compared to the general interstellar medium, we carry out similar fitting to $\mathrm{HCO}^{+} J=4-3$ and $\mathrm{H}^{13} \mathrm{CO}^{+} J=4-3$ observations.

Results. ALMA images show centrally peaked extended emission from $\mathrm{HCO}^{+}$and $\mathrm{H}^{13} \mathrm{CO}^{+}$. $\mathrm{DCO}^{+}$emission lies in a resolved ring from $\sim 110$ to $160 \mathrm{AU}$. The outer radius approximately corresponds to the size of the CO snowline as measured by previous lower resolution observations of $\mathrm{CO}$ lines in this disk. The $\mathrm{ALMA} \mathrm{DCO}^{+}$data now resolve and image the $\mathrm{CO}$ snowline directly.

Conclusions. In the best fitting models, $\mathrm{HCO}^{+}$exists in a region extending from the $19 \mathrm{~K}$ isotherm to the photodissociation layer with an abundance of $3 \times 10^{-10}$ relative to $\mathrm{H}_{2}$. DCO ${ }^{+}$exists within the $19-21 \mathrm{~K}$ region of the disk with an abundance ratio $\left[\mathrm{DCO}^{+}\right] /\left[\mathrm{HCO}^{+}\right]=0.3$. This represents a factor of $10^{4}$ enhancement of the $\mathrm{DCO}^{+}$abundance within this narrow region of the HD 163296 disk. Such a high enhancement has only previously been seen in prestellar cores. The inferred abundances provide a lower limit to the ionization fraction in the midplane of the cold outer disk $\left(\gtrsim 4 \times 10^{-10}\right)$, and suggest the utility of $\mathrm{DCO}^{+}$as a tracer of its parent molecule $\mathrm{H}_{2} \mathrm{D}^{+}$.

Key words. stars: pre-main sequence - protoplanetary disks - submillimeter: stars - stars: individual: HD 163296

\section{Introduction}

Circumstellar disks of gas and dust are the environments in which planets form, as well as many molecules which may be transported to planets. Understanding the density and temperature structure of these disks, in particular the dense midplane, is necessary to understand the dynamic and chemical processes involved (Williams \& Cieza 2011). However, directly probing

\footnotetext{
* Appendix $\mathrm{A}$ is available in electronic form at http: //WwW . aanda.org
}

the midplane is difficult. Many of the most abundant molecules, such as $\mathrm{CO}$ and $\mathrm{HCO}^{+}$, are present throughout much of the disk but have high opacity. Optically thin isotopologues such as $\mathrm{C}^{18} \mathrm{O}$ or $\mathrm{H}^{13} \mathrm{CO}^{+}$may have detectable emission from the midplane, but it is confused by emission from higher layers. In addition, much of the midplane becomes unobservable as $\mathrm{CO}$ and other tracers freeze onto grains due to low temperatures at large radii.

$\mathrm{DCO}^{+}$has been suggested as an excellent tracer of the disk midplane near the freezeout region of $\mathrm{CO}$ because the decline in temperature provides an ideal environment for gas-phase $\mathrm{DCO}^{+}$ formation. It is formed in the gas phase primarily by the transfer 
of a deuterium atom from the $\mathrm{H}_{2} \mathrm{D}^{+}$ion to a $\mathrm{CO}$ molecule (Wootten 1987).

Chemical models predict the enhanced formation of the parent molecule $\mathrm{H}_{2} \mathrm{D}^{+}$at low temperatures from the reaction

$\mathrm{H}_{3}^{+}+\mathrm{HD} \Leftrightarrow \mathrm{H}_{2} \mathrm{D}^{+}+\mathrm{H}_{2}+\Delta E$,

where $\Delta E \sim 220 \mathrm{~K}$ (Roberts \& Millar 2000). This effect is further enhanced by the reduction in the $\mathrm{H}_{2}$ ortho/para ratio at low temperatures, where the energy difference between the ortho ground state $(J=1)$ and para ground state $(J=0)$ otherwise provides internal energy for the back reaction $(\Delta E \sim 170 \mathrm{~K}$, Maret \& Bergin 2007; Pagani et al. 2009).

The parent molecule of $\mathrm{H}_{2} \mathrm{D}^{+}, \mathrm{H}_{3}^{+}$, is effectively destroyed by CO (Jørgensen et al. 2004), which can remain in the gas phase down to a temperature of $\sim 20 \mathrm{~K}$. Thus a reduction in the $\mathrm{CO}$ abundance will make $\mathrm{HD}$ the preferred destroyer of $\mathrm{H}_{3}^{+}$and lead to an enhancement of $\mathrm{H}_{2} \mathrm{D}^{+}$. The increase in $\mathrm{H}_{2} \mathrm{D}^{+}$formation, in turn, is expected to enhance the formation of $\mathrm{DCO}^{+}$.

However, $\mathrm{CO}$ is also one of the parent molecules of $\mathrm{DCO}^{+}$, so while $\mathrm{DCO}^{+}$formation is aided by a decrease in $\mathrm{CO}$ abundance, it will also be constrained at temperatures where too little $\mathrm{CO}$ remains in the gas phase. A balance between low temperatures and $\mathrm{CO}$ abundance is required to enhance $\mathrm{DCO}^{+}$abundance.

These factors have led to models predicting that the $\mathrm{DCO}^{+}$ abundance will be enhanced in a narrow range of temperatures of a few Kelvin near the $\mathrm{CO}$ freezeout temperature of $\sim 20 \mathrm{~K}$, which successfully explain emission from pre-stellar cores (e.g., Caselli et al. 1999; Pagani et al. 2012). In disks, line emission from $\mathrm{DCO}^{+}$is then expected to trace the rise in formation of its parent molecule, $\mathrm{H}_{2} \mathrm{D}^{+}$at increasing radii. $\mathrm{H}_{2} \mathrm{D}^{+}$serves as the basis of many deuteration processes, and is one of the principal ions in cold dense gas. $\mathrm{DCO}^{+}$emission will not rise indefinitely, instead it will form a ring which is truncated at the midplane $\sim 20 \mathrm{~K}$ isotherm, the $\mathrm{CO}$ "snowline" where the gas phase $\mathrm{CO}$ abundance drops due to freezeout onto grains (e.g., Aikawa et al. 2002; Willacy 2007). Tracing the CO snowline is of importance to theories of planet formation because of the increase in the surface density of solids which can accelerate the growth of larger bodies.

While ions such as $\mathrm{HCO}^{+}$and $\mathrm{DCO}^{+}$are expected to trace low temperature conditions within the disk, they can also provide limits on the ionization fraction (e.g., Öberg et al. 2011b), a key parameter governing accretion in the disk. At ionization fractions $\left(x_{\text {ion }}\right)$ less than $10^{-13}$, the magneto-rotational instability that drives viscous accretion breaks down due to a lack of conductivity. This creates "dead zones" (Gammie 1996), which in turn provide one mechanism of enhancing planet formation by the generation of high pressure vortices that trap large dust grains, thus increasing grain growth efficiency (e.g., Barge \& Sommeria 1995; Varnière \& Tagger 2006). Direct assessment of the ionization fraction will serve to test recently proposed mechanisms by which cosmic rays, one of the primary ionization mechanisms, might be excluded from a circumstellar disk by the young star's stellar winds and magnetic field (Cleeves et al. 2013).

Single dish observations have previously revealed emission from $\mathrm{DCO}^{+}$towards two T Tauri stars, TW Hya (van Dishoeck et al. 2003; Guilloteau et al. 2006), with interferometric observations suggesting a ringlike morphology for $\mathrm{DCO}^{+}$in TW Hya (Qi et al. 2008; Öberg et al. 2012). Comparison with $\mathrm{HCO}^{+}$ has indicated a disk averaged $\mathrm{DCO}^{+} / \mathrm{HCO}^{+}$abundance ratio of $\sim 0.04$ (TW Hya, van Dishoeck et al. 2003), which is a factor of 1000 higher than the interstellar medium $[\mathrm{D}] /[\mathrm{H}]$ abundance of $\approx 10^{-5}$ (Watson 1976). Even higher abundances have been seen in dark clouds such as $\mathrm{L} 1544\left(\mathrm{DCO}^{+} / \mathrm{HCO}^{+}=0.12\right.$, Caselli et al. 1999). Later observations with the Sub-Millimeter Array (SMA) have detected $\mathrm{DCO}^{+}$in several additional disks, though not with sufficient resolution to see the details of the morphology (Öberg et al. 2010, 2011a,b).

Nearby gas-rich disks provide ideal sites for examination of the cold disk midplane using $\mathrm{DCO}^{+}$. One such system is the Herbig Ae star HD $163296(\alpha=17 \mathrm{~h} 56 \mathrm{~m} 21.29 \mathrm{~s}, \delta=$ $-21^{\circ} 57^{\prime} 21^{\prime \prime} 87$, J2000, spectral type A1, Houk \& Smith-Moore 1988), which is relatively nearby (122 pc, Perryman et al. 1997) and young (4 Myr, van den Ancker et al. 1998). It has long served as a prototype for studies of gas and dust rich protoplanetary disks (e.g., Mannings \& Sargent 1997). Previous studies have determined that it has a relatively massive disk $\left(0.07-0.08 M_{\odot}\right)$ and a gas-to-dust ratio from 100 to 150 (e.g., Qi et al. 2011; Tilling et al. 2012). The disk is observed at an inclination of $44^{\circ}$, and position angle of $\sim 133^{\circ}$ east-of-north. Due to its high luminosity $\left(\sim 40 L_{\odot}\right)$, the $20 \mathrm{~K}$ region is at a large radius which can be resolved with science verification data from the Atacama Large Millimeter/submillimeter Array (ALMA). The massive disk has a high total line-of-sight column density of $\mathrm{DCO}^{+}$and the intermediate inclination means that vertical and radial spatial information can be obtained, as well as kinematic information.

Qi et al. (2011, henceforth Q11) measured the radius of the CO snowline in the disk around HD 163296 by fitting high spectral resolution visibility data from the SMA with models of ${ }^{13} \mathrm{CO}$ emission incorporating a sharp drop in abundance as a function of distance from the star. They estimated that the $\mathrm{CO}$ snowline of HD 163296 lies at a distance of 155 AU, corresponding to a midplane temperature of $19 \mathrm{~K}$ in their physical model.

In this paper, we present resolved imaging of $\mathrm{DCO}^{+}$line emission from HD 163296 and examine the utility of $\mathrm{DCO}^{+}$as a tracer of disk structure. The paper is structured as follows. In Sect. 2, we describe the observations and data reduction, and present our results in Sect. 3. We compare the disk properties to models in Sect. 4 and explore the implications for further disk studies in Sect. 5. We summarize our findings in Sect. 6.

\section{Observations}

Band 7 observations of HD 163296 were carried out on June 9, 11, 22, and July 6, 2012 for a total on-source time of 59.4 minutes as part of the ALMA science verification program 2011.0.000010.SV. Median precipitable water vapor levels (pwv) were 1.8, 0.7, 0.3, and $0.7 \mathrm{~mm}$, respectively. The ALMA array included between 17 and $2012 \mathrm{~m}$ diameter antennas and baselines from $13 \mathrm{~m}$ to $402 \mathrm{~m}$. The four correlator bands were centered on the $\mathrm{CO} J=3-2, \mathrm{HCO}^{+} J=4-3, \mathrm{H}^{13} \mathrm{CO}^{+} J=4-3$, and $\mathrm{DCO}^{+} J=5-4$ transitions at $345.796,356.734,346.998$, and $360.170 \mathrm{GHz}$, with channel widths of 122.1, 122.1, 244.1, and $30.5 \mathrm{kHz}$, and total bandwidths of 468.8, 468.8, 937.5, and 117.2 MHz, respectively. For gain, bandpass, and flux calibration, we used J1733-130, J1924-292, and Neptune, respectively.

We used the Common Astronomy Software Applications package (CASA, McMullin et al. 2007) to carry out calibration and imaging of the visibility datasets. Self-calibration was carried out for both phases and amplitudes using line free channels. We used the CLEAN algorithm with natural weighting and circular clean masks with diameters of $\sim 9^{\prime \prime}, 5^{\prime \prime}$, and $5^{\prime \prime}$ to produce channel maps of the $\mathrm{HCO}^{+}, \mathrm{H}^{13} \mathrm{CO}^{+}$, and $\mathrm{DCO}^{+}$lines, respectively. The image cubes have a velocity resolution of $0.4 \mathrm{~km} \mathrm{~s}^{-1}$ 

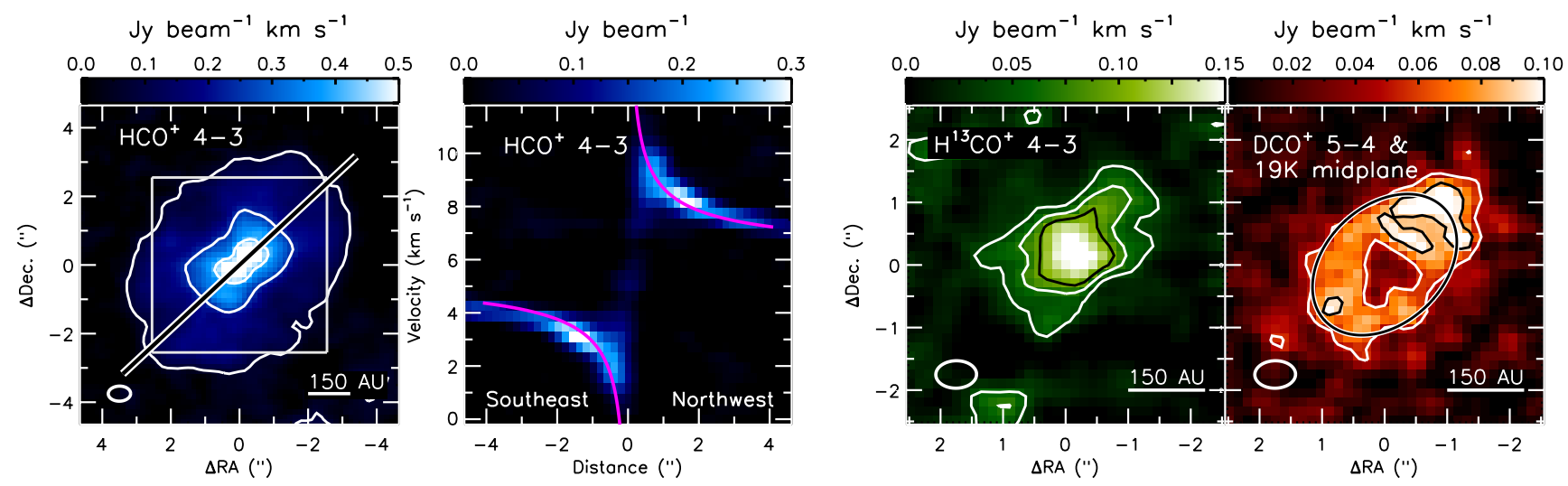

Fig. 1. Left: integrated emission map of $\mathrm{HCO}^{+}$(blue) from $0-12 \mathrm{~km} \mathrm{~s}^{-1}$ with contours showing 3, 10, and $17 \sigma$ emission $(\sigma=$ $25 \mathrm{mJy}$ beam ${ }^{-1} \mathrm{~km} \mathrm{~s}^{-1}$ ). The white-bordered black line shows the positions from which we constructed the position-velocity diagram (middle-left), and the gray box shows the smaller region for which we show $\mathrm{H}^{13} \mathrm{CO}^{+}$and $\mathrm{DCO}^{+}$line emission (middle-right and right). Middle-left: the $\mathrm{HCO}^{+}$ position-velocity diagram. The magenta curves show the Keplerian velocity profile for an assumed stellar mass of $2.3 M_{\odot}$, disk inclination of $44^{\circ}$, and systemic velocity of $5.8 \mathrm{~km} \mathrm{~s}^{-1}$. Middle-right: integrated emission map of $\mathrm{H}^{13} \mathrm{CO}^{+}$(green) from $0.8-10.4 \mathrm{~km} \mathrm{~s}^{-1}$ with contours showing 3,5 , and $7 \sigma$ emission $\left(\sigma=14 \mathrm{mJy}_{\text {beam }}^{-1} \mathrm{~km} \mathrm{~s}^{-1}\right)$. Right: integrated emission map of $\mathrm{DCO}^{+}$(red) from $0.8-10.4 \mathrm{~km} \mathrm{~s}^{-1}$ with contours showing 3 and $5 \sigma$ emission $\left(\sigma=18 \mathrm{mJy}_{\text {beam }}^{-1} \mathrm{~km} \mathrm{~s}^{-1}\right)$. The $19 \mathrm{~K}$ contour of the midplane temperature at a radius of 155 AU in the model disk is overlaid on the $\mathrm{DCO}^{+}$emission using a white-bordered black ellipse. In the maps of integrated emission, the $\sim 0{ }^{\prime} 65 \times 00^{\prime} 44$ beam is shown in the lower left, while a scale is shown in the lower right.

and pixel size of 0 ' $^{\prime} 15$ in each data cube. The synthesized beam size is $\sim 00^{\prime} 65 \times 00^{\prime} 44$, at a position angle of $\sim 90^{\circ}$.

In this paper we examine the emission from $\mathrm{HCO}^{+}$and its isotopologues, with a focus on $\mathrm{DCO}^{+}$. Discussion of the $\mathrm{CO}$ line emission from the disk may be found in de Gregorio-Monsalvo et al. (2013) and in Chapillon et al. (in prep.), while Klaassen et al. (2013), discuss CO line emission associated with the disk wind.

\section{Results}

In Table 1, we list the rms noise limits found in map regions and channels showing no emission, as well as the spatially and spectrally integrated emission in each line. We also present the line fluxes of our adopted model (discussed below). Line emission is detected at velocities between 0 and $12 \mathrm{~km} \mathrm{~s}^{-1}$ in $\mathrm{HCO}^{+}$, and at velocities of 0.8 to $10.4 \mathrm{~km} \mathrm{~s}^{-1}$ in $\mathrm{H}^{13} \mathrm{CO}^{+}$and $\mathrm{DCO}^{+}$. All three lines have a central LSR (local standard of rest) velocity of $5.8 \mathrm{~km} \mathrm{~s}^{-1}$, consistent with previous observations (e.g., Mannings \& Sargent 1997, Q11). In Fig. 1, we show the integrated maps of the emission from all three isotopologues in the velocity ranges given above.

\subsection{Morphology}

The $\mathrm{HCO}^{+}$emission exhibits a smooth spatial profile that peaks at the stellar position. We fit an ellipse to the $3 \sigma$ contour of the integrated emission map, and find that it has major and minor axes of $7 . .8$ and $5 . .3$ and a position angle of $133^{\circ}$ east of north. Under the assumption of a flat disk these correspond to a disk with an outer radius of $475 \mathrm{AU}$ observed at an inclination of $47^{\circ}$, comparable to previous inclination determinations. The position-velocity diagram in Fig. 1 shows that the line-of-sight velocities along the disk major axis exhibit a Keplerian velocity profile.

$\mathrm{H}^{13} \mathrm{CO}^{+}$emission is only detected near the center of the disk, with the $2 \sigma$ contour extending 1 ". 4 from the disk center (170 AU).
Table 1. Observational and modeling results.

\begin{tabular}{|c|c|c|c|}
\hline Line & $\begin{array}{c}\text { RMS } \\
\left(\mathrm{mJy} \mathrm{beam}^{-1}\right)\end{array}$ & $\begin{array}{c}\text { Flux } \\
\left(\mathrm{Jy} \mathrm{km} \mathrm{s}^{-1}\right)\end{array}$ & $\begin{array}{l}\text { Model flux } \\
\left(\mathrm{Jy} \mathrm{km} \mathrm{s}^{-1}\right)\end{array}$ \\
\hline $\mathrm{HCO}^{+}$ & 11.3 & $18.7 \pm 0.7$ & 26.0 \\
\hline $\mathrm{H}^{13} \mathrm{CO}^{+} J=4-3$ & 8.2 & $1.0 \pm 0.2$ & 1.4 \\
\hline $\mathrm{DCO}^{+} \quad J=5-4$ & 13.1 & $2.2 \pm 0.4$ & 1.7 \\
\hline
\end{tabular}

Notes. Each species and transition is given along with the rms in emission-free channels with a velocity width of $0.4 \mathrm{~km} \mathrm{~s}^{-1}$. The third and fourth columns show the line flux integrated over the disk, and the flux of the adopted model.

$\mathrm{DCO}^{+}$emission is not detected at the stellar position but is confined to an annulus in the integrated emission map with outer major and minor axes of 3 .' 3 and 2 .'2, and inner major and minor axes of 1.'4 and 1'. 0 . This suggests $\mathrm{DCO}^{+}$emission originates in a ring with a central radius of $\sim 140 \mathrm{AU}$ and a width less than $110 \mathrm{AU}$ (i.e. extending from a radius of at least $95 \mathrm{AU}$ to less than $195 \mathrm{AU})$. This approximately corresponds to the CO snowline found by Q11 from lower resolution SMA observations of $\mathrm{CO}$ and its isotopologues, a relation we explore in more detail below. We show the midplane $19 \mathrm{~K}$ contour at a radius of $155 \mathrm{AU}$ from our adopted model (see Sect. 4) in Fig. 1. There is an apparent peak in emission to the northwest. However, in this work we address the large scale structure, and do not attempt to model variations within that structure.

\subsection{Estimated column densities}

$\mathrm{H}^{13} \mathrm{CO}^{+}$is expected to have an abundance a factor of 75 less than $\mathrm{HCO}^{+}$, corresponding to the ${ }^{12} \mathrm{C} /{ }^{13} \mathrm{C}$ abundance ratio in the interstellar medium (Frerking et al. 1982). We assume that the $\mathrm{H}^{13} \mathrm{CO}^{+} J=4-3$ line is optically thin and can be used to estimate the median molecular column density in the disk. The $\mathrm{H}^{13} \mathrm{CO}^{+} J=4-3 / \mathrm{HCO}^{+} J=4-3$ intensity ratio for positions and velocities where $\mathrm{H}^{13} \mathrm{CO}^{+}$is detected has a median value of 0.18 , confirming the high optical depth of $\mathrm{HCO}^{+}$with $\tau \approx 16$ under the assumption of local thermodynamic equilibrium (LTE). 
We use the online RADEX 1D non-LTE radiative transfer tool $^{1}$ (van der Tak et al. 2007) to make an initial estimate of the column density. Assuming a kinetic temperature $T_{\mathrm{K}}=40 \mathrm{~K}$ and gas density $n_{\mathrm{H}_{2}}=10^{7} \mathrm{~cm}^{-3}$ (intermediate values from the disk model of Q11), and assuming $\Delta V=1 \mathrm{~km} \mathrm{~s}^{-1}$, we adjust the $\mathrm{HCO}^{+}$and $\mathrm{H}^{13} \mathrm{CO}^{+}$column densities until the model intensity ratios match the median observed intensity ratio. We find line of sight column densities of $2.1 \times 10^{14} \mathrm{~cm}^{-2}$ and $2.8 \times 10^{12} \mathrm{~cm}^{-2}$ for $\mathrm{HCO}^{+}$and $\mathrm{H}^{13} \mathrm{CO}^{+}$, respectively. Correcting for the disk inclination, these are vertical column densities of $1.5 \times 10^{14} \mathrm{~cm}^{-2}$ and $2.0 \times 10^{12} \mathrm{~cm}^{-2}$

The column density sensitivity of our observations is estimated using RADEX, as well. For each transition, we convert the $3 \sigma$ limits in our maps of integrated line emission to antenna temperature. We then assume gas densities and temperatures typical of the expected emission regions. For $\mathrm{HCO}^{+}$and $\mathrm{H}^{13} \mathrm{CO}^{+}$, we assume the same $T_{\mathrm{K}}$ and $n_{\mathrm{H}_{2}}$ values as above, and for $\mathrm{DCO}^{+}$ we assume $n_{\mathrm{H}_{2}}=10^{9} \mathrm{~cm}^{-3}$ and $T_{\mathrm{K}}=19 \mathrm{~K}$ (values from the midplane $\mathrm{CO}$ snowline of Q11). Our integrated maps have approximate column density sensitivities of $1.7 \times 10^{12}, 0.7 \times 10^{12}$, and $2.5 \times 10^{12} \mathrm{~cm}^{-2}$ for $\mathrm{HCO}^{+}, \mathrm{H}^{13} \mathrm{CO}^{+}$, and $\mathrm{DCO}^{+}$, respectively. These estimates do not reflect the variation in density and temperature across the regions in which these molecules exist, factors that require more detailed radiative transfer modeling as described in the following section.

\section{Modeling}

\subsection{Physical structure}

Analysis of the $\mathrm{DCO}^{+}$spatial distribution requires a physical model which specifies the temperature and gas density as a function of both radius and height in the disk. We use a parametric disk model to approximate the best-fit model of Q11, with parameters adjusted to fit the spectral energy distribution and line fluxes for $\mathrm{CO}$ and its isotopologues in the literature. The details of the density and temperature structure of our disk model, as well as our radiative transfer models and ray-tracing, can be found in Appendix A. Simulated observations of our models, mimicking the $(u, v)$ coverage of the data, were carried out using the built-in simulation tasks of CASA.

We used this spectral energy distribution (SED) -constrained model as the basis for constructing a grid of $\mathrm{HCO}^{+}, \mathrm{H}^{13} \mathrm{CO}^{+}$, and $\mathrm{DCO}^{+}$models, with energy levels, transition frequencies, and Einstein A coefficients taken from the Leiden Atomic and Molecular Database $^{2}$ (Schöier et al. 2005). We manually varied the isotopologue abundances, and in the case of $\mathrm{DCO}^{+}$the region in which it is abundant (discussed below), and compared the resulting channel maps to the observations.

\subsection{Molecular abundances}

The $\mathrm{HCO}^{+}$and $\mathrm{H}^{13} \mathrm{CO}^{+}$abundances $\left(\chi_{\mathrm{HCO}^{+}}\right.$and $\chi_{\mathrm{H}^{13} \mathrm{CO}^{+}}$, respectively) are taken to be constant within the region populated by the parent $\mathrm{CO}$ molecule. This region is bounded on the upper side by photodissociation (i.e., below the height where a hydrogen column density of $N_{\mathrm{H}_{2}}=2 \times 10^{21} \mathrm{~cm}^{-2}$ is reached, adopted from the translucent-cloud modeling of Visser et al. 2009) and on the lower side by the $19 \mathrm{~K} \mathrm{CO}$ freezeout zone (Q11). Outside this region we treat the abundance as zero. We explored $\mathrm{HCO}^{+}$

\footnotetext{
1 http://www.sron.rug.nl/ vdtak/radex/radex.php

2 http://home.strw.leidenuniv.nl/ moldata/
}

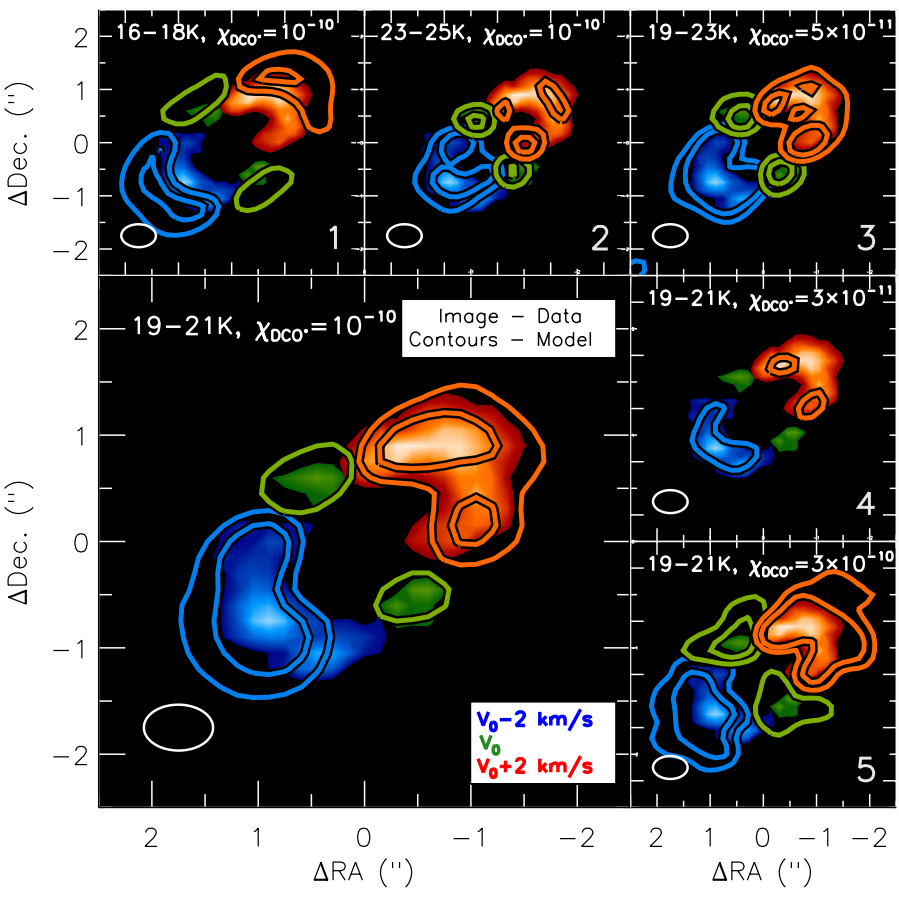

Fig. 2. Comparison of integrated intensities of our $\mathrm{DCO}^{+}$models (contours, 3 and $5 \sigma, \sigma=15 \mathrm{mJy}^{-1} \mathrm{bem}^{-1} \mathrm{~km} \mathrm{~s}^{-1}$ ) to the observations (image, colors show intensities from $3 \sigma$ ). Colors indicate the velocities included in constructing the zeroth moment maps. Clockwise from the upper left, small figures show: 1) decreasing $T_{\text {low, } \mathrm{DCO}^{+}}$and $T_{\text {high, } \mathrm{DCO}}$ by $3 \mathrm{~K}, 2$ ) raising $T_{\text {low }, \mathrm{DCO}^{+}}$and $T_{\mathrm{high}, \mathrm{DCO}^{+}}$by $4 \mathrm{~K}, 3$ ) broadening the temperature range from $2 \mathrm{~K}$ to $4 \mathrm{~K}$, with $\chi_{\mathrm{DCO}^{+}}$halved to maintain the same total $\mathrm{DCO}^{+} J=5-4$ line flux, 4) reduction of $\chi_{\mathrm{DCO}^{+}}$by a factor of 3 , and 5) raising $\chi_{\mathrm{DCO}^{+}}$by a factor of 3 .

abundances relative to $\mathrm{H}_{2}$ ranging from $10^{-11}$ to $10^{-5}$ in logarithmic steps of 0.5 (corresponding to factors $\sim 3$ ) and setting the $\mathrm{H}^{13} \mathrm{CO}^{+}$abundance to $1 / 75$ that of $\mathrm{HCO}^{+}$. We find a best simultaneous match to the $\mathrm{HCO}^{+}$and $\mathrm{H}^{13} \mathrm{CO}^{+}$integrated intensities and channel maps with a $\mathrm{HCO}^{+}$abundance of $3 \times 10^{-10}$, and a corresponding $\mathrm{H}^{13} \mathrm{CO}^{+}$abundance of $4 \times 10^{-12}$. These constant abundances are adopted throughout this work.

We carried out a similar manual fit to the $\mathrm{DCO}^{+} J=5-4$ emission. Our $\mathrm{DCO}^{+}$models include the same assumption of a uniform abundance $\left(\chi_{\mathrm{DCO}^{+}}\right)$within the region of $\mathrm{DCO}^{+}$enhancement, and an abundance of zero outside of this region. However, the region in which $\mathrm{DCO}^{+}$is enhanced is defined by both high temperature and low temperature cutoffs, $T_{\text {high,DCO }}{ }^{+}$ and $T_{\text {low, } \mathrm{DCO}^{+}}$. Above $T_{\text {high, } \mathrm{DCO}^{+}}, \mathrm{DCO}^{+}$cannot form in large quantities due to the destruction of $\mathrm{H}_{3}^{+}$and $\mathrm{H}_{2} \mathrm{D}^{+}$by gas phase $\mathrm{CO}$ and ortho- $\mathrm{H}_{2}$, respectively. Below $T_{\text {low, } \mathrm{DCO}^{+}}$it cannot form due to a lack of gas phase $\mathrm{CO}$. We find that a $\chi_{\mathrm{DCO}^{+}}$of $1 \times 10^{-10}$ at temperatures between $T_{\mathrm{high}, \mathrm{DCO}^{+}}=21 \mathrm{~K}$ and $T_{\mathrm{low} \mathrm{DCO}^{+}}=19 \mathrm{~K}$ provides the best fit to both the total flux and to channel maps. We compare fluxes from our adopted model to the observed fluxes in Table 1.

Figure 2 presents the comparison between our best fit $\mathrm{DCO}^{+}$ model and the observations in the large lower left panel, for $2 \mathrm{~km} \mathrm{~s}^{-1}$ wide channel maps centered at 4,6 , and $8 \mathrm{~km} \mathrm{~s}^{-1}$, respectively. We simultaneously display these maps in blue, green, and red scaled uniformly above $3 \sigma$, and overlay the 3 and $5 \sigma$ model contours in the same velocity ranges (in these broad channel maps, $\sigma=15$ mJy beam ${ }^{-1} \mathrm{~km} \mathrm{~s}^{-1}$ ). 


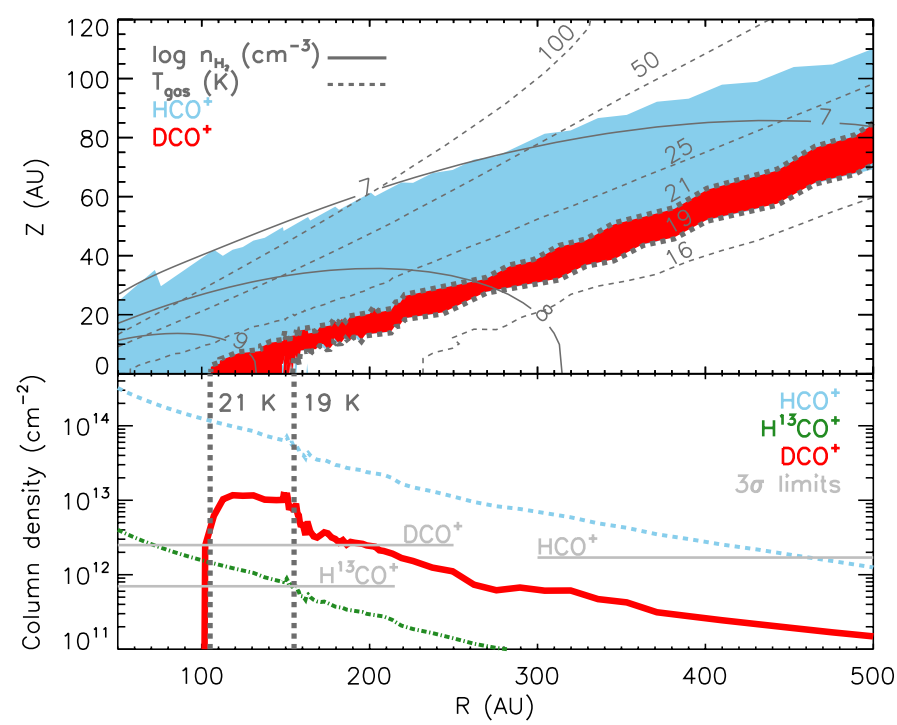

Fig. 3. Top: regions of non-zero $\mathrm{HCO}^{+}$(blue) and $\mathrm{DCO}^{+}$(red) abundance, overlaid with contours indicating the hydrogen density (solid gray lines) and temperature (dashed gray lines). The 19 and $21 \mathrm{~K}$ contours are shown with a thick dark gray line. The region containing $\mathrm{HCO}^{+}$is bounded on the upper and lower sides by $\mathrm{CO}$ photodissociation and by freezeout at $19 \mathrm{~K}$, respectively. The $\mathrm{DCO}^{+}$region is bounded by the temperature range 19-21 K. Bottom: the column density of $\mathrm{HCO}^{+}$and its isotopologues (colored lines), with the $19 \mathrm{~K} \mathrm{CO}$ snowline indicated by the thick dark gray dashed line. The broad plateau of the $\mathrm{DCO}^{+}$column density from $\sim 110-150$ AU explains the ring morphology seen in line emission. The $3 \sigma$ line sensitivities (from Sect. 3.2) are shown with the horizontal gray lines.

The five small panels of Fig. 2 show how the emission varies if we change the temperature range and abundance of $\mathrm{DCO}^{+}$. The panels show: 1) if we decrease the temperature bounds by $3 \mathrm{~K}$ or 2) increase them by $4 \mathrm{~K}, 3$ ) if we broaden the temperature range by a factor of two (while halving the abundance to maintain approximately the same integrated emission), or if we 4) decrease the abundance by a factor of 3 , or 5) increase it by a factor of 3 . These effects highlight the relation between disk properties and observables: the temperature range in which $\mathrm{DCO}^{+}$has high abundance primarily sets the observed spatial region of emission, while the abundance adjusts the strength of that emission.

In Fig. 3, we illustrate the $\mathrm{HCO}^{+}$and $\mathrm{DCO}^{+}$regions of our adopted model and the molecular column density as a function of radius in the disk. Due to the increasing physical thickness of the $\mathrm{DCO}^{+}$region, decreasing gas density, and our assumption of a constant abundance, the $\mathrm{DCO}^{+}$column density is relatively constant from 110 to $150 \mathrm{AU}$, corresponding to the brightest region of observed emission.

\section{Discussion}

\subsection{Why a ring?}

The data constrain $\mathrm{DCO}^{+}$emission to a narrow ring near the previously inferred $\mathrm{CO}$ snowline, and our models constrain the physical parameters of this region. Three factors combine to restrict the detection of $\mathrm{DCO}^{+}$to a narrow ring:

1. As the temperature decreases to $21 \mathrm{~K}$, the $\mathrm{CO}$ abundance begins to drop due to freezeout and allows the effective formation of one of the parent molecules of $\mathrm{DCO}^{+}, \mathrm{H}_{2} \mathrm{D}^{+}$. In addition, the ortho/para ratio of $\mathrm{H}_{2}$ drops to $\sim 10^{-3}$, inhibiting the back reaction of $\mathrm{H}_{2} \mathrm{D}^{+}$with $\mathrm{H}_{2}$. These low temperatures are first reached at a radius of $\sim 110 \mathrm{AU}$, and the $\mathrm{DCO}^{+}$column density will rise as its abundance becomes enhanced (Fig. 3, $21 \mathrm{~K}$ contour).

2. Where the temperature drops below $19 \mathrm{~K}$ (i.e. at radii greater than $155 \mathrm{AU}$ in the midplane), the other parent molecule of $\mathrm{DCO}^{+}, \mathrm{CO}$, will be largely removed from the gas phase due to freezeout. This will in turn reduce the $\mathrm{DCO}^{+}$column density (Fig. 3, $19 \mathrm{~K}$ contour). The temperature bounded region is present at larger radii above the midplane, but at these heights, $\mathrm{DCO}^{+}$emission faces a third constraint.

3. The gas density, $n_{\mathrm{H}_{2}}$, decreases with height above the midplane, in turn reducing the column density of $\mathrm{DCO}^{+}$. This is illustrated by comparing the top and bottom panels of Fig. 3 . The column density decreases at the $\mathrm{CO}$ snowline at $155 \mathrm{AU}$ (bottom, vertical dashed gray line). This is the radius where the $19 \mathrm{~K}$ temperature contour rises above the midplane (top, thick dashed gray line). As the $19 \mathrm{~K}$ isotherm rises in the disk, the gas density, and the corresponding column densities, decrease (top, solid contours).

In all of these aspects, the $\mathrm{DCO}^{+}$chemistry in the HD 163296 disk is consistent with the chemistry used to explain the emission from deuterated molecules in dark clouds and prestellar cores.

At low optical depths and nearly constant temperature, the integrated intensity will be an approximately linear function of column density. We can infer from Fig. 3 that the outer edge of the broad peak of $\mathrm{DCO}^{+}$emission traces the $\mathrm{CO}$ snowline in the midplane, while emission at larger radii traces a layer of constant temperature that rises from the midplane of the disk. Under our enrichment scenario, the inner edge indicates where the formation rate of $\mathrm{H}_{2} \mathrm{D}^{+}$rises.

In our model, the column density of $\mathrm{DCO}^{+}$drops by a factor of $\sim 4$ from 150 to $200 \mathrm{AU}$. In the current observations, the peak intensity is $6 \sigma$, suggesting that only a factor of 3 improvement in sensitivity is needed to probe this disk structure to a radius of $\sim 200$ AU. With twice as many antennas, the full ALMA array will be able to accomplish such an observation with only $\sim 2 \mathrm{~h}$ integration time.

\subsection{Probing the vertical structure}

Models with a factor of 3 increase in column density - which could be achieved either by increasing the relative abundance or the overall gas density - lead to much larger structures being detectable in the model images (see panels 4 and 5 of Fig. 2). This is due to an increase in the column density at larger radii in the disk. As this is also the region where the $19 \mathrm{~K}$ isotherm rises from the midplane, this suggests the utility of $\mathrm{DCO}^{+}$not just as a probe of the regions of enhanced $\mathrm{H}_{2} \mathrm{D}^{+}$formation and the midplane $\mathrm{CO}$ snowline, but also with higher sensitivity observations a probe of the vertical disk structure.

In combination with detailed chemical models, this provides constraints on the degree of vertical mixing, as $\mathrm{DCO}^{+}$that moves upward from this region will be destroyed, and that which moves down in the disk will freeze out on grains.

\subsection{Sensitivity to $T_{\text {high }}$ and $T_{\text {low }}$}

At a radius of $155 \mathrm{AU}$, the disk midplane temperature changes at a rate of $\approx 1 \mathrm{~K}$ per $20 \mathrm{AU}$. Small changes in the modeled 
$\mathrm{DCO}^{+}$temperature range then correspond to large changes in the physical emission region. The resolution of the beam, 0 ' $^{\prime} 44$, corresponds to about $50 \mathrm{AU}$ at the distance of HD 163296, therefore differences of $2.5 \mathrm{~K}$ in $T_{\text {high,DCO }}+$ and $T_{\text {low,DCO}}+$ will lead to resolvable differences in the radial distribution of line emission. The center of the temperature range can decrease by less than $3 \mathrm{~K}$ and increase by less than $4 \mathrm{~K}$ (see panels 1 and 2 of Fig. 2). The width of the temperature range in which $\mathrm{DCO}^{+}$exists cannot be much larger than $2 \mathrm{~K}$, as the ring of emission becomes too wide (see panel 3 of Fig. 2). Changes in the position of emission in the disk will also lead to changes in the line of sight velocity, with the velocity changing on a scale of $\approx 0.2 \mathrm{~km} \mathrm{~s}^{-1}$ per $20 \mathrm{AU} . T_{\mathrm{high}_{\mathrm{DCO}}+}$ and $T_{\text {low,DCO}}+$ cannot shift far from $\sim 20 \mathrm{~K}$ as the ring both changes in size and the emission shifts to different velocities.

\subsection{Enhancement of $\mathrm{DCO}^{+}$}

While $\mathrm{DCO}^{+}$is restricted to a much smaller vertical area of the disk than $\mathrm{HCO}^{+}$, the regions in which these ions are abundant overlap near the $\mathrm{CO}$ snowline. Under our assumption of a uniform $\mathrm{HCO}^{+}$abundance within the region from the $\mathrm{CO}$ snowline to the CO photodissociation limit at $N_{\mathrm{H}_{2}}=2 \times 10^{21} \mathrm{~cm}^{-2}$, the relative abundance of $\mathrm{DCO}^{+}$to $\mathrm{HCO}^{+}$is as high as 0.3 within the sub-region which lies near $20 \mathrm{~K}$. This represents a local enhancement by a factor of at least $10^{4}$ over the ISM $[\mathrm{D}] /[\mathrm{H}]$ ratio of $\approx 10^{-5}$ (Watson 1976), and is a higher deuteration than that observed in pre-stellar cores where CO is also frozen out (e.g., Caselli et al. 1999). Some models of vertical chemical structure predict that the $\mathrm{HCO}^{+}$abundance begins declining above the region of enhanced $\mathrm{DCO}^{+}$abundance (Aikawa \& Nomura 2006). In such a scenario, the local $\mathrm{DCO}^{+}$to $\mathrm{HCO}^{+}$abundance ratio would be even higher. While $\mathrm{H}^{13} \mathrm{CO}$ observations could provide constraints on this scenario, distentangling the midplane emission from that in higher layers in the disk requires more detailed modeling of the $\mathrm{HCO}^{+}$abundance structure and higher signalto-noise $\mathrm{H}^{13} \mathrm{CO}^{+}$data.

In the images presented here, $\mathrm{DCO}^{+}$emission covers approximately $1 / 4$ of the observed $\mathrm{HCO}^{+}$emission area (Fig. 1). In our model, the vertical thickness of the $\mathrm{DCO}^{+}$region is also approximately $1 / 4$ that of the $\mathrm{HCO}^{+}$region (Fig. 3). Taking both geometric effects into account, the disk averaged abundance ratio is approximately 0.02 , comparable to the disk-averaged abundance ratio found in TW Hya (0.04, van Dishoeck et al. 2003).

\subsection{Ionization fraction}

The model $\mathrm{HCO}^{+}$and $\mathrm{DCO}^{+}$abundances provide a lower limit to the ionization fraction of the disk. In the warm molecular disk where $\mathrm{HCO}^{+}$is present, the ionization fraction must be at least $3 \times 10^{-10}$. Assuming that it continues to be present into the cold midplane region where the $\mathrm{DCO}^{+}$abundance is enhanced (as it is in our simple model), the minimum ionization fraction would be $4 \times 10^{-10}\left(\mathrm{HCO}^{+}+\mathrm{DCO}^{+}\right)$. This is at least an order of magnitude higher than that found in the cool molecular layer of DM Tau described by Öberg et al. (2011b). This is also three orders of magnitude higher than the critical ionization fraction at which dead zones form. Observations of other region specific ions (e.g., $\mathrm{H}_{2} \mathrm{D}^{+}$outside the $\mathrm{CO}$ snowline, Öberg et al. 2011b) will allow for estimation of the importance of this mechanism for planet formation throughout the disk.

\subsection{Comparison to other disks}

The scenario we have modeled here for HD 163296 is consistent with that described for DM Tau by Öberg et al. (2011b), wherein they find that unresolved $\mathrm{DCO}^{+}$emission can be fit using an enhanced $\mathrm{DCO}^{+}$abundance in the temperature range of 16-20 K. This temperature range is slightly lower and broader than that found here.

$\mathrm{DCO}^{+} J=3-2$ emission has been mapped towards TW Hya by Qi et al. (2008) and can also be fit by a ring-like morphology. They simulate a three-layer disk structure by assuming a model in which the molecular column density is a function of distance from the star, and the abundance at any given radius is constant within the middle layer. They fit a power law radial column density profile for each molecule in their study, as well as a vertical structure defined relative to the hydrogen column density. Under this model, they find that $\mathrm{DCO}^{+}$emission is best fit by either a model in which its column density increases away from the star with a sharp truncation at $90 \mathrm{AU}$, or by a two-part power law function which increases to $70 \mathrm{AU}$, and then decreases. In both models, the $\mathrm{DCO}^{+}$exists in a layer extending from hydrogen column densities of 0.1 to 10 times $1.59 \times 10^{21} \mathrm{~cm}^{-2}$, the "disk surface". This region has temperatures ranging from $\sim 16-50 \mathrm{~K}$, and densities from $\sim 10^{7}-10^{8} \mathrm{~cm}^{-3}$. Midplane temperatures at this radius are $\sim 10 \mathrm{~K}$.

The $19 \mathrm{~K}$ midplane isotherm in TW Hya lies at $30 \mathrm{AU}$ in the Qi et al. (2008) model. $\mathrm{DCO}^{+}$in this disk therefore does not appear to trace the same isotherm as in HD 163296 and in pre-stellar cores. Either some mechanism acts to inhibit the low temperature enhancement of $\mathrm{DCO}^{+}$at $30 \mathrm{AU}$, or alternatively, some process generates a second peak in $\mathrm{DCO}^{+}$around $70 \mathrm{AU}$ as seen by the SMA.

One possibility is related to the formation of $\mathrm{H}_{2} \mathrm{D}^{+}$, the $\mathrm{DCO}^{+}$parent molecule. Whereas we have focused on the reduction of $\mathrm{CO}$ abundance allowing for greater formation of $\mathrm{H}_{2} \mathrm{D}^{+}$ from $\mathrm{H}_{3}^{+}$, other molecules can serve to inhibit its formation (therefore inhibiting its reaction products, such as $\mathrm{DCO}^{+}$). $\mathrm{H}_{3}^{+}$ can also react with $\mathrm{N}_{2}$. Perhaps the $70 \mathrm{AU}$ radius in TW Hya traces $\mathrm{N}_{2}$ freezeout which occurs at slightly lower temperatures (Bisschop et al. 2006) resulting in a second peak in $\mathrm{H}_{2} \mathrm{D}^{+}$. If some residual $\mathrm{CO}$ was present, due to mixing from warmer layers or release from grains, then $\mathrm{DCO}^{+}$could form.

Alternatively, the underlying model of gas and dust density and temperature could be in need of revision. Recent SMA continuum observations (Andrews et al. 2012) indicate the mmemitting dust disk is truncated at 60 AU. This would have far reaching effects on the thermal structure and disk chemistry, including the potential release of $\mathrm{CO}$ into the gas phase due to increased penetration of ultraviolet radiation (an effect noted by Andrews et al.). This could allow the gas-phase formation of $\mathrm{DCO}^{+}$even outside the $\mathrm{CO}$ snowline.

At $30 \mathrm{AU}$, it is also possible that the ortho-to-para ratio of $\mathrm{H}_{2}$ has not dropped to the low levels of $\sim 10^{-3}$ expected for these low midplane temperatures and required to enhance $\mathrm{H}_{2} \mathrm{D}^{+}$abundance. The ortho-to-para conversion is governed by the $\mathrm{H}^{+}+$ ortho- $\mathrm{H}_{2} \rightarrow \mathrm{H}^{+}+$para- $\mathrm{H}_{2}$ reaction, which has a timescale that is proportional to the $\mathrm{H}^{+}$abundance. For an $\mathrm{H}^{+}$abundance of at most $10^{-12}$ with respect to $\mathrm{H}_{2}$ (Walsh et al. 2012) the timescale is at least $3 \times 10^{5} \mathrm{yr}$ for a density of $10^{9} \mathrm{~cm}^{-3}$ and rate coefficient of $1.1 \times 10^{-10} \mathrm{~cm}^{3} \mathrm{~s}^{-1}$ at $10 \mathrm{~K}$ (Pagani et al. 2013). If some high temperature event (e.g., a recent flare) has reset the $\mathrm{H}_{2}$ ortho/para ratio to a high temperature value in the inner disk temporarily, it may not have equilibrated back to the low value needed to boost the $\mathrm{H}_{2} \mathrm{D}^{+}$abundance at the $30 \mathrm{AU}$ radius. Note 
that the ortho- $\mathrm{H}_{2} \mathrm{~S}(1)$ line at $17 \mu \mathrm{m}$ has been detected toward TW Hya (Najita et al. 2010) and interpreted to arise from radii out to $30 \mathrm{AU}$ (Gorti et al. 2011). Even though the mid-infrared emission arises from the warm surface layers, some of this ortho$\mathrm{H}_{2}$ may have been mixed down to the midplane.

A superthermal abundance of ortho- $\mathrm{H}_{2}$ also has other chemical consequences, most notably in driving the reaction $\mathrm{N}^{+}+$ ortho- $\mathrm{H}_{2} \rightarrow \mathrm{NH}^{+}+\mathrm{H}$, which triggers the nitrogen chemistry leading to $\mathrm{NH}_{3}$. The strong Herschel-HIFI detection of $\mathrm{NH}_{3}$ in the TW Hya disk (Hogerheijde et al., in prep.) would be consistent with this scenario.

Ultimately, this discussion points to the need for high spatial resolution ALMA mapping of $\mathrm{DCO}^{+}$and other molecules sensitive to CO freezeout (e.g., Qi et al. 2013a) in the TW Hya and other disks to determine the relative importance of the $\mathrm{H}_{2} \mathrm{D}^{+}$ enhancement versus $\mathrm{CO}$ freeze-out.

\subsection{Dependence on physical models}

Here we relate the observed emission to physical/chemical properties via an adopted structure model for the disk. This model has been constrained via observations that trace various regions of the disk (e.g., the SED and CO observations). CO observations in particular are primarily sensitive to a thin surface layer between the height where photodissociation of $\mathrm{CO}$ no longer effectively operates and the height where the lines become optically thick. Even the emission of optically thin isotopologues of $\mathrm{CO}$ are strongly affected by the emission from these surface layers, and only partially probe the disk midplane. Because our $\mathrm{DCO}^{+}$observations are thought to probe the disk midplane, it is appropriate to investigate the dependence of our results on the details of the adopted physical model.

Compared to our model, which is based on the work of Q11, the best-fit disk structure of de Gregorio-Monsalvo et al. (2013) has 1-3 K lower temperatures and factor $\sim 1.5$ higher densities in the 100-150 AU midplane region. Using this model, we find that our observations are best described if $\mathrm{DCO}^{+}$is enhanced between temperatures of 16 and $20 \mathrm{~K}$ (compared to 19-21 K found using the Q11 model). The best fit $\mathrm{DCO}^{+}$abundance is $\sim 7 \times 10^{-11}$, compared to $1 \times 10^{-10}$ for the Q11 model. These higher densities affect the $\mathrm{H}^{13} \mathrm{CO}^{+}$abundance in a similar fashion, leading to an $\mathrm{HCO}^{+}$abundance of $\sim 2 \times 10^{-10}$. The differences in gas density affect the molecular abundance in the same manner, leading to a similar localized $\mathrm{DCO}^{+} / \mathrm{HCO}^{+}$ratio of $\sim 0.3$.

Although the details of the best-fit model clearly depend on the adopted disk structure, the general characteristics remain unchanged: $\mathrm{DCO}^{+}$is a sensitive tracer of the narrow temperature range where $\mathrm{CO}$ freezes out and $\mathrm{H}_{2} \mathrm{D}^{+}$starts to increase in abundance. Other high-resolution observations of species that also trace this transition (e.g., $\mathrm{N}_{2} \mathrm{H}^{+}$; see Qi et al. 2013b) will further constrain the parameter range of allowed models.

\section{Conclusions}

We have presented ALMA Science Verification images of the $\mathrm{HCO}^{+} J=4-3, \mathrm{H}^{13} \mathrm{CO}^{+} J=4-3$, and $\mathrm{DCO}^{+} J=5-4$ emission from the HD 163296 circumstellar disk. While $\mathrm{HCO}^{+}$traces the entire disk and is detected at the stellar position, the $\mathrm{DCO}^{+}$ emission is seen in a ring tracing the region between the rise of $\mathrm{H}_{2} \mathrm{D}^{+}$formation and the $\mathrm{CO}$ snowline. Using radiative transfer modeling, we have shown that the emission can be matched by a model in which $\mathrm{DCO}^{+}$has high abundance within a limited temperature range near the $\mathrm{CO}$ freezeout temperature (from 19 to $21 \mathrm{~K}$ ). This emission traces the previously determined $\mathrm{CO}$ snowline. Comparison of the modeled abundances indicates a $\left[\mathrm{DCO}^{+}\right] /\left[\mathrm{HCO}^{+}\right]$ratio of 0.3 , the highest yet observed. This in turn suggests that gas phase deuteration processes can lead to local enhancements of the abundance of $\mathrm{DCO}^{+}$on the order of a factor of $10^{4}$, comparable to that observed in some dark clouds. The high abundance of these ions also indicates that the ionization fraction must be $\gtrsim 10^{-10}$ in the cold midplane, three orders of magnitude higher than necessary to maintain the MHD turbulence that powers viscous accretion.

Future high sensitivity, high resolution observations with ALMA will allow for the direct imaging of many chemical processes in disks. These studies will provide important constraints on models of disk physical structure even in the vertical direction. In conjunction with tracers of the disk surface (e.g. CO) and the bulk gas (e.g. $\mathrm{C}^{18} \mathrm{O}$ ), we are entering the era of "disk tomography" where disks can be mapped in a region-by-region fashion. This will, in turn, allow us to study chemistry and dynamics as they vary throughout the disk.

Acknowledgements. This paper makes use of the following ALMA data: ADS/JAO.ALMA\#2011.0.00010.SV. ALMA is a partnership of ESO (representing its member states), NSF (USA) and NINS (Japan), together with NRC (Canada) and NSC and ASIAA (Taiwan), in cooperation with the Republic of Chile. The Joint ALMA Observatory is operated by ESO, AUI/NRAO and NAOJ. Astrochemistry in Leiden is supported by NOVA, KNAW and EU A-ERC grant 291141 CHEMPLAN. D.H. is additionally supported by SRON. IdGM acknowledges the Spanish MICINN grant AYA2011-30228-C03 (co-funded with FEDER funds). A.H. acknowledges support from the Millennium Science Initiative, Chilean Ministry of Economy: Nucleus P10-022-F. The National Radio Astronomy Observatory is a facility of the National Science Foundation operated under cooperative agreement by Associated Universities, Inc.

\section{References}

Aikawa, Y., \& Nomura, H. 2006, ApJ, 642, 1152

Aikawa, Y., van Zadelhoff, G. J., van Dishoeck, E. F., \& Herbst, E. 2002, A\&A, 386,622

Andrews, S. M., Wilner, D. J., Hughes, A. M., Qi, C., \& Dullemond, C. P. 2009, ApJ, 700, 1502

Andrews, S. M., Wilner, D. J., Hughes, A. M., et al. 2012, ApJ, 744, 162

Barge, P., \& Sommeria, J. 1995, A\&A, 295, L1

Bisschop, S. E., Fraser, H. J., Öberg, K. I., van Dishoeck, E. F., \& Schlemmer, S. 2006, A\&A, 449, 1297

Brinch, C., \& Hogerheijde, M. R. 2010, A\&A, 523, A25

Caselli, P., Walmsley, C. M., Tafalla, M., Dore, L., \& Myers, P. C. 1999, ApJ, 523, L165

Cleeves, L., Adams, F. C., \& Bergin, E. A. 2013, ApJ, 772, 5

Cutri, R. M., Wright, E. L., Conrow, T., et al. 2011, Explanatory Supplement to the WISE Preliminary Data Release Products, Tech. Rep.

de Gregorio-Monsalvo, I., Ménard, F., Dent, W., et al. 2013, A\&A,

DOI: $10.1051 / \ldots / 201321603$

Draine, B. T. 2006, ApJ, 636, 1114

Dullemond, C. P., \& Dominik, C. 2004, A\&A, 417, 159

Egan, M. P., Price, S. D., Kraemer, K. E., et al. 2003, VizieR Online Data Catalog: V/114

Frerking, M. A., Langer, W. D., \& Wilson, R. W. 1982, ApJ, 262, 590

Gammie, C. F. 1996, ApJ, 457, 355

Gorti, U., Hollenbach, D., Najita, J., \& Pascucci, I. 2011, ApJ, 735, 90

Guilloteau, S., Piétu, V., Dutrey, A., \& Guélin, M. 2006, A\&A, 448, L5

Hartmann, L., Calvet, N., Gullbring, E., \& D'Alessio, P. 1998, ApJ, 495, 385

Helou, G., \& Walker, D. W. 1988, in Infrared astronomical satellite (IRAS) catalogs and atlases, The small scale structure catalog, 7

Houk, N., \& Smith-Moore, M. 1988, Michigan Catalogue of Two-dimensional Spectral Types for the HD Stars, Declinations $-26^{\circ} .0$ to $-12^{\circ} .0,4$ (Ann Arbor: Univ. Michigan)

Ishihara, D., Onaka, T., Kataza, H., et al. 2010, A\&A, 514, A1 
Jørgensen, J. K, Schöier, F. L., \& van Dishoeck, E. F. 2004, A\&A, 416, 603 Klaassen, P. D., Juhasz, A., Mathews, G. S., et al. 2013, A\&A, 555, A73

Mannings, V., \& Sargent, A. I. 1997, ApJ, 490, 792

Maret, S., \& Bergin, E. A. 2007, ApJ, 664, 956

McMullin, J. P., Waters, B., Schiebel, D., Young, W., \& Golap, K. 2007, in Astronomical Data Analysis Software and Systems XVI, eds. R. A. Shaw,

F. Hill, \& D. J. Bell, ASP Conf. Ser., 376, 127

Najita, J. R., Carr, J. S., Strom, S. E., et al. 2010, ApJ, 712, 274

Öberg, K. I., Qi, C., Fogel, J. K. J., et al. 2010, ApJ, 720, 480

Öberg, K. I., Qi, C., Fogel, J. K. J., et al. 2011a, ApJ, 734, 98

Öberg, K. I., Qi, C., Wilner, D. J., \& Andrews, S. M. 2011b, ApJ, 743, 152

Öberg, K. I., Qi, C., Wilner, D. J., \& Hogerheijde, M. R. 2012, ApJ, 749, 162

Pagani, L., Vastel, C., Hugo, E., et al. 2009, A\&A, 494, 623

Pagani, L., Bourgoin, A., \& Lique, F. 2012, A\&A, 548, L4

Pagani, L., Lesaffre, P., Jorfi, M., et al. 2013, A\&A, 551, A38

Perryman, M. A. C., Lindegren, L., Kovalevsky, J., et al. 1997, A\&A, 323, L49

Qi, C., Wilner, D. J., Aikawa, Y., Blake, G. A., \& Hogerheijde, M. R. 2008, ApJ, 681,1396

Qi, C., D’Alessio, P., Öberg, K. I., et al. 2011, ApJ, 740, 84
Qi, C., Öberg, K. I., \& Wilner, D. J. 2013a, ApJ, 765, 34

Qi, C., Öberg, K. I., Wilner, D. J., et al. 2013b, Science, 341, 630

Roberts, H., \& Millar, T. J. 2000, A\&A, 361, 388

Schöier, F. L., van der Tak, F. F. S., van Dishoeck, E. F., \& Black, J. H. 2005, A\&A, 432, 369

Tilling, I., Woitke, P., Meeus, G., et al. 2012, A\&A, 538, A20

van den Ancker, M. E., de Winter, D., \& Tjin A Djie, H. R. E. 1998, A\&A, 330, 145

van der Tak, F. F. S., Black, J. H., Schöier, F. L., Jansen, D. J., \& van Dishoeck, E. F. 2007, A\&A, 468, 627

van Dishoeck, E. F., Thi, W.-F., \& van Zadelhoff, G.-J. 2003, A\&A, 400, L1

Varnière, P., \& Tagger, M. 2006, A\&A, 446, L13

Visser, R., van Dishoeck, E. F., \& Black, J. H. 2009, A\&A, 503, 323

Walsh, C., Nomura, H., Millar, T. J., \& Aikawa, Y. 2012, ApJ, 747, 114

Watson, W. D. 1976, Rev. Mod. Phys., 48, 513

Willacy, K. 2007, ApJ, 660, 441

Williams, J. P., \& Cieza, L. A. 2011, ARA\&A, 49, 67

Wootten, A. 1987, in Astrochemistry, eds. M. S. Vardya, \& S. P. Tarafdar, IAU Symp., 120, 311

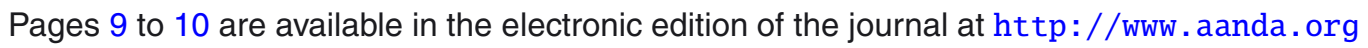


G. S. Mathews et al.: ALMA imaging of the CO snowline of the HD 163296 disk with DCO ${ }^{+}$

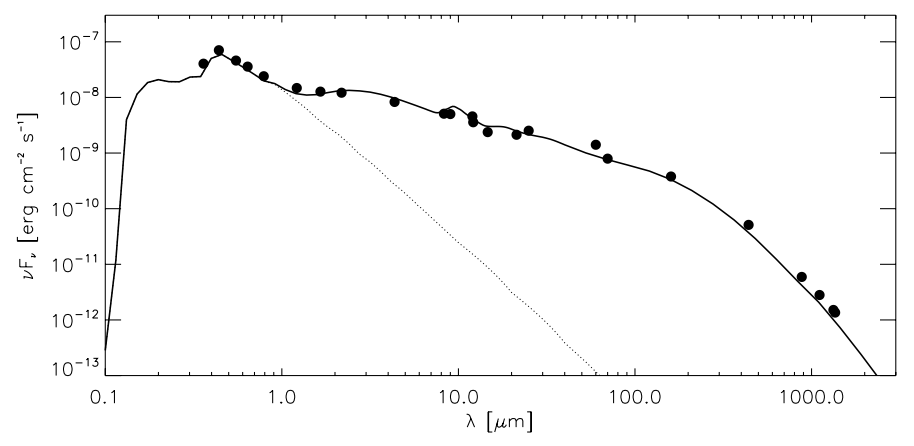

Fig. A.1. Spectral energy distribution (black line) of our best fit model, compared to dereddened photometry $\left(A_{V}=0.3\right.$, Q11) from the literature (Q11, Tilling et al. 2012; Cutri et al. 2011; Ishihara et al. 2010; Egan et al. 2003; Helou \& Walker 1988). The stellar photosphere is shown as the thin dashed line.

Table A.1. Comparison of observed and model fluxes.

\begin{tabular}{llcc}
\hline \hline Property & $\begin{array}{c}\text { Model } \\
\left(\mathrm{Jy} \mathrm{km} \mathrm{s}^{-1}\right)\end{array}$ & $\begin{array}{c}\text { Observed } \\
\left(\mathrm{Jy} \mathrm{km} \mathrm{s}^{-1}\right)\end{array}$ \\
\hline $\mathrm{CO}$ & $J=2-1$ & 51 & $54.17 \pm 0.39^{a}$ \\
${ }^{13} \mathrm{CO}$ & $J=2-1$ & 21 & $18.76 \pm 0.24^{a}$ \\
$\mathrm{C}^{18} \mathrm{O}$ & $J=2-1$ & 9 & $6.30 \pm 0.16^{a}$ \\
$\mathrm{CO}$ & $J=3-2$ & 74 & $98.72 \pm 1.69^{a}$ \\
$\mathrm{C}^{17} \mathrm{O}$ & $J=3-2$ & 7 & $11.64 \pm 0.76^{a}$ \\
$\mathrm{HCO}^{+}$ & $J=4-3$ & 26.0 & $18.7 \pm 0.7$ \\
$\mathrm{H}^{13} \mathrm{CO}^{+}$ & $J=4-3$ & 1.4 & $1.0 \pm 0.2$ \\
$\mathrm{DCO}^{+}$ & $J=5-4$ & 1.7 & $2.2 \pm 0.4$ \\
\hline
\end{tabular}

Notes. ${ }^{(a)}$ Observed fluxes from Q11.

\section{Appendix A: Modeling details}

\section{A.1. Parametric dust and gas structure}

We adopt the overall disk properties derived by Q11 from fitting the broadband spectral energy distribution and spatial extent of their mm data: a disk mass $M_{\text {disk }}=0.089 M_{\odot}$, with an inner edge $R_{\text {in }}=0.6 \mathrm{AU}$ and a critical radius $R_{\mathrm{C}}=150 \mathrm{AU}$. We define the surface density as (e.g. Andrews et al. 2009):

$\Sigma(R)=\Sigma_{\mathrm{C}}\left(\frac{R}{R_{\mathrm{C}}}\right)^{-\gamma} \exp \left[-\left(\frac{R}{R_{\mathrm{C}}}\right)^{2-\gamma}\right]$,

where we set the surface density power law index, $\gamma$, equal to 1 , the value for a self-similar accretion disk in steady-state (Hartmann et al. 1998). We adopt the gas-to-dust ratio of 154 from Q11 as well as their two component dust grain population (small and large) used to simulate dust settling. Both populations are made of a $60 \% / 40 \%$ mix of astronomical silicates and graphite, and follow a power law grain size distribution $n(a) \propto a^{-3.5}$ (Draine 2006) with a minimum grain size $a_{\min }=0.005 \mu \mathrm{m}$ for both populations. The small grain population has a maximum grain size $a_{\text {max,small }}=0.25 \mu \mathrm{m}$, and the large population has $a_{\text {max,large }}=1 \mathrm{~mm}$. The small grain component is set to include $1.6 \%$ of the dust mass $\left(f_{\text {small }}=0.016\right)$.

The vertical distribution is modeled as a Gaussian with angular scale height

$h=h_{\mathrm{C}}\left(\frac{R}{R_{\mathrm{C}}}\right)^{\psi}$,
Table A.2. Adopted model.

\begin{tabular}{|c|c|c|}
\hline Property & Value & $\begin{array}{l}\text { Explored range } \\
\text { or reference }\end{array}$ \\
\hline $\begin{array}{l}\text { Stellar parameters } \\
M_{\text {star }} \\
R_{\text {star }} \\
T_{\text {star }} \\
\end{array}$ & $\begin{array}{r}2.3 M_{\odot} \\
2.0 R_{\odot} \\
9333 \mathrm{~K} \\
\end{array}$ & $\begin{array}{l}\text { Q11 } \\
\text { Q11 } \\
\text { Q11 } \\
\end{array}$ \\
\hline $\begin{array}{l}\text { Dust parameters } \\
\text { [silicate]/[graphite] } \\
a_{\text {min }} \\
a_{\text {max,small }} \\
a_{\text {max,large }} \\
f_{\text {small }} \\
\end{array}$ & $\begin{array}{c}60 \% / 40 \% \\
0.005 \mu \mathrm{m} \\
0.25 \mu \mathrm{m} \\
1 \mathrm{~mm} \\
0.016 \\
\end{array}$ & $\begin{array}{l}\text { Q11 } \\
\text { Q11 } \\
\text { Q11 } \\
\text { Q11 } \\
\text { Q11 } \\
\end{array}$ \\
\hline $\begin{array}{l}\text { Disk parameters } \\
M_{\text {disk }} \\
\text { gas/dust } \\
R_{\text {in }} \\
R_{\mathrm{C}} \\
h_{\text {puffedrim }} \\
\gamma \\
\psi \\
\text { Inclination } \\
\text { Position angle } \\
\text { Systemic velocity } \\
f \text { (Small grains) } \\
h_{\text {small }} \\
h_{\text {large }} \\
h_{\text {main }} \\
h_{\text {tail }} \\
f_{\text {tail }} \\
\end{array}$ & $\begin{array}{c}0.089 M_{\odot} \\
154 \\
0.6 \mathrm{AU} \\
150 \mathrm{AU} \\
1.67 \\
1 \\
0.066 \\
44^{\circ} \\
133^{\circ} \\
5.8 \mathrm{~km} \mathrm{~s}^{-1} \\
0.016 \\
0.08 \\
0.06 \\
0.10 \\
0.20 \\
0.05 \\
\end{array}$ & $\begin{array}{c}\text { Q11 } \\
\text { Q11 } \\
\text { Q11 } \\
\text { Q11 } \\
\text { Q11 } \\
\text { Hartmann et al. (1998) } \\
\text { Tilling et al. (2012) } \\
\text { Q11 } \\
\text { Q11 } \\
\text { Q11 } \\
\text { Q11 } \\
0.04-0.20 \\
0.04-0.20 \\
0.04-0.24 \\
0.04-0.24 \\
0.00-0.20 \\
\end{array}$ \\
\hline $\begin{array}{l}\text { Molecule parameters } \\
\chi_{\mathrm{CO}} \\
\chi_{\mathrm{HCO}^{+}} \\
\chi_{\mathrm{H}^{13} \mathrm{CO}^{+}} \\
\chi_{\mathrm{DCO}^{+}} \\
T_{\mathrm{high}_{\mathrm{DCO}}+} \\
T_{\text {low }, \mathrm{DCO}^{+}} \\
\end{array}$ & $\begin{array}{c}10^{-4} \\
3 \times 10^{-10} \\
\chi_{\mathrm{HCO}^{+} / 75} \\
1 \times 10^{-10} \\
21 \mathrm{~K} \\
19 \mathrm{~K} \\
\end{array}$ & $\begin{array}{c}\text { Frerking et al. (1982) } \\
10^{-11}-10^{-5} \\
\text { Frerking et al. (1982) } \\
10^{-11}-10^{-8} \\
19-25 \mathrm{~K} \\
16-21 \mathrm{~K} \\
\end{array}$ \\
\hline
\end{tabular}

where $h_{\mathrm{C}}$ is the angular scale height at the characteristic radius $R_{\mathrm{C}}$ and $\psi$ describes the power-law disk flaring. We set $\psi=0.066$, for consistency with the modeling of Tilling et al. (2012). The two dust component populations have independent scale heights $\left(h_{\mathrm{C}}\right)$ of $h_{\text {small }}$ and $h_{\text {large }}$, respectively, which we adjust to match the spectral energy distribution (discussed below).

The gas density distribution produced by the physical model of Q11 is approximately Gaussian at low heights and has a long "tail" to large heights (cf. their Fig. 7). In order to approximate this, we have constructed the gas density distribution using a two-component model with independently varying scale heights for the main component ( $\left.h_{\text {main }}\right)$ and the lower mass, larger scale height tail $\left(h_{\text {tail }}\right)$. In addition, we vary the distribution of gas mass between these two components, with a term $f_{\text {tail }}$ describing the fraction of the total gas mass in the tail.

\section{A.2. Determining the disk parameters}

With this framework in place, we adjust parameters to match the SED and CO line fluxes in the literature for HD 163296. We carried out continuum radiative transfer modeling using the 2D code RADMC (Dullemond \& Dominik 2004), which receives as input stellar properties and a dust density structure and outputs the resulting temperature structure. It includes a raytracing code, Raytran, for producing the model spectral energy 
distribution. We adjusted $h_{\text {small }}$ and $h_{\text {large }}$ in order to approximate the temperature structure found in Q11 (cf. their Fig. 9), which came from a self-consistent vertical structure calculation. We made further small adjustments to these parameters in order to match the SED. Our adopted disk model has small- and large-grain scale heights of 0.08 and 0.06 , respectively, and we show the resulting SED in Fig. A.1. In our further modeling, we assume the gas temperature matches the mean dust temperature calculated in this modeling, which is a reasonable approximation in the dense regions upon which we focus.

After setting the dust parameters, we adjust $h_{\text {main }}, h_{\text {tail }}$, and $f_{\text {tail }}$ to approximate the gas density structure found in Q11 (their Fig. 9). We fine-tune these parameters by comparing our modeled fluxes of $\mathrm{CO}$ and its isotopologues with the observed fluxes of Q11, focusing on the low- $J$ rotational lines that are expected to come largely from the cool outer disk regions studied here. We assume a relative $\mathrm{CO}$ abundance $[\mathrm{CO}] /[\mathrm{H}] \approx 10^{-4}$ (Frerking et al. 1982), which drops to zero at hydrogen column densities less than $2 \times 10^{21} \mathrm{~cm}^{-2}$ due to photodissociation (Visser et al. 2009) and at temperatures less than $19 \mathrm{~K}$ due to freezeout (Qi et al. 2011). We also assume a ratio of ${ }^{12} \mathrm{C}$ to ${ }^{13} \mathrm{C}$ of 75 , and ratios of ${ }^{16} \mathrm{O}$ to ${ }^{18} \mathrm{O}$ and ${ }^{17} \mathrm{O}$ of 500 and 1750 , respectively (Frerking et al. 1982). To generate our model line emission, we input our adopted dust density and temperature structure, along with the model gas density structure, to the line radiative transfer code LIME (Brinch \& Hogerheijde 2010), and we achieve a match to the observed line fluxes within $\sim 50 \%$.

The gas "tail" has a scale height $h_{\text {tail }}=0.2$ and includes $5 \%$ of the gas mass $\left(f_{\text {tail }}\right)$, while the "main" gas component has a scale height $h_{\text {main }}=0.1$. In Table A.1, we list the integrated fluxes of the $\mathrm{CO}$ lines which we use to determine the disk gas structure. Table A.2 lists and categorizes all disk modeling parameters, as well as the range of explored values or the reference from which the fixed value was adopted. In both tables, we repeat the $\mathrm{HCO}^{+}, \mathrm{H}^{13} \mathrm{CO}^{+}$, and $\mathrm{DCO}^{+}$properties discussed in the main body of the text. 